\title{
Effektivität von Sauerstoff- Demand-Systemen bei Patienten mit chronisch obstruktiver Atemwegserkrankung
}

\begin{abstract}
Zusammenfassung: Um die Mobilität von Patienten unter Sauerstoff-Langzeittherapie zu optimieren, sind tragbare Sauerstoffsysteme verfügbar. Diese haben aufgrund ihres begrenzten Sauerstoffvorrates eine eingeschränkte Reichweite. Um diese zu vergrößern und die Kosten der Sauerstoffbehandlung zu senken, werden Sauerstoff-Demandsysteme (DODS) benutzt. Ziel dieser Arbeit war es, die Effektivität von drei DODS (DOC2000 [D], TransTracheal Inc.; Oxytron [O], Weinmann; Pulsair [P], DeVilbiss, Sunrise Medical) im Vergleich zu kontinuierlicher Sauerstoffgabe (CONT) zu bewerten. 17 Patienten mit schwerer Atemwegsobstruktion bei COPD (Alter 67,82 $\pm 9,46$ Jahre, FEV $1,23 \pm 0,69 \mathrm{I}, \mathrm{PaO}_{2} 48,8 \pm 6,7 \mathrm{~mm} \mathrm{Hg}$ ) wurden untersucht. Die verschiedenen Sauerstoffsysteme (sowohl CONT als auch DODS) wurden den Patienten für je $\mathbf{3 0}$ Minuten in randomisierter Reihenfolge mit einem Fluss von 2 Litern pro Minute appliziert. Bei jedem System wurden nach jeweils 15 und 30 Minuten Anwendungszeit Blutgasanalysen durchgeführt. Die Sauerstoffsättigung wurde kontinuierlich gemessen. Nach 15 Minuten wurde kein signifikanter Unterschied des arteriellen Sauerstoffpartialdrucks zwischen CONT und den Demandsystemen festgestellt. Nach 30 Minuten jedoch wurden signifikant erniedrigte $\mathrm{PaO}_{2}$-Werte im Vergleich zu CONT gefunden für $\mathrm{O}$ und $\mathrm{D}$ $(p<0,01)$. Kein signifikanter Unterschied war zwischen den $\mathrm{PaO}_{2}$-Werten für CONT und $\mathrm{P}$ zu sehen. Um für eine Messung der Verbesserung der Reichweite vergleichbare Bedingungen zu schaffen, wurde die Flussrate bei $O$ und $D$ so erhöht, dass gleiche $\mathrm{PaO}_{2}$-Werte im Vergleich zu CONT erreicht wurden. Damit konnte die Reichweite einer definierten Sauerstoffmenge mit $P$ um 161,5 Prozent und mit $O$ um 172 Prozent vergrößert werden, während sich unter Verwendung von D lediglich eine Reichweitenvergrößerung von 17,2 Prozent erzielen ließ. Eine Zunahme der Reichweite von tragbaren Sauerstoffsystemen kann durch Verwendung von Sauerstoff-Demandsystemen erreicht werden ohne ein Absinken des arteriellen Sauerstoffpartialdrucks und somit ohne Qualitätseinbußen bei der Sauerstoffbehandlung. Jedoch gibt es Unterschiede in der Effektivität der einzelnen Demandsysteme.
\end{abstract}

Efficacy of Demand Oxygen Delivery Systems in Patients with COPD: To maintain mobility in patients with chronic hypoxemia who are under long-term oxygen therapy, portable oxygen systems are available. They have the disadvantage of a short range. To prolong the range and to reduce the cost of

Pneumologie 2001; 55: $306-310$

(c) Georg Thieme Verlag Stuttgart · New York ISSN 0934-8387

\author{
U. Johann ${ }^{1}$, J. Fichter ${ }^{2}$, G. W. Sybrecht ${ }^{1}$ \\ ${ }^{1}$ Universitätskliniken des Saarlandes, Innere Medizin V \\ (Prof. Dr. G. W. Sybrecht) Homburg/Saar \\ 2 Paracelsus-Klinik Osnabrück
}

oxygen treatment, demand oxygen delivery systems (DODS) are used. Aim of the study was to compare three DODS (DOC2000 [D], TransTracheal Inc.; Oxytron [O], Weinmann; Pulsair [P], DeVilbiss, Sunrise Medical) with continuous oxygen delivery. 17 patients (age $67.82 \pm 9.46$ years; $\mathrm{FEV}_{1} 1.23 \pm 0.69$ I; $\mathrm{PaO}_{2}$ $48.8 \pm 6.7 \mathrm{~mm} \mathrm{Hg}$ ) were studied. The continuous flow oxygen (CONT) and the DODS were applied to the patients for 30 minutes each in random sequence with an airflow of $21 / \mathrm{min}$. After 15 and 30 minutes arterial blood gas analysis was done. Oxygen saturation was recorded continuously by pulseoximetry. After 15 minutes no significant differences in $\mathrm{PaO}_{2}$ were found between CONT and DODS. After 30 minutes no significant difference in $\mathrm{PaO}_{2}$ was found in CONT as compared to P. Significant lower $\mathrm{PaO}_{2}$ values were found for $\mathrm{O}$ and $\mathrm{D}$ as compared to CONT $(p<0.01)$. With $P$ the range of a portable oxygen source was increased by 161.5 percent, with 0 by 172 percent, with $D$ the range was increased by only 17.2 percent. Prolongation of range of a portable oxygen source can be achieved by means of DODS without a decrease of $\mathrm{PaO}_{2}$ and thus without loss of quality of the oxygen treatment. However, there are differences in efficacy between the DODS.

\section{Einleitung}

Sauerstoff-Demandsysteme werden eingesetzt um den Sauerstoffverbrauch und damit auch die Kosten einer SauerstoffLangzeittherapie zu senken. Es handelt sich dabei um inspirationsgetriggerte Geräte, die über einen empfindlichen, elektronischen Drucksensor den frühinspiratorischen, subatmosphärischen Druck messen. Wird der Druck subatmosphärisch, so wird für kurze Zeit ein Ventil geöffnet und ein SauerstoffBolus abgegeben. Spätinspiratorisch und während der Expiration bleibt das Ventil geschlossen. Für eine maximale Effektivität muss der Bolus möglichst früh während der Inspirationsphase freigesetzt werden, das heißt, die Triggerempfindlichkeit muss hoch und die Latenzzeit gering sein. Mit Hilfe von Demandsystemen lassen sich bis zu 86 Prozent Sauerstoff einsparen [1]. Systematische Vergleiche von Sauerstoff-Demandsystemen verschiedener Hersteller wurden bislang jedoch kaum durchgeführt.

In dieser Arbeit werden drei Sauerstoff-Demandsysteme auf die Effektivität im Vergleich zur Gabe von kontinuierlichem Sauerstoff untersucht. Zielparameter waren der arterielle Sauerstoffpartialdruck und die Sauerstoffsättigung. Zusätzlich werden der Spareffekt der einzelnen Demandsysteme und die 
dadurch entstehende Reichweitenvergrößerung einer gegebenen Sauerstoffmenge gemessen.

\section{Methode}

Es wurden 17 Patienten mit chronisch obstruktiver Atemwegserkrankung untersucht. Es handelte sich dabei um 2 Frauen und 15 Männer im Alter von 42 bis 81 Jahren (mittleres Alter 67,8 $\pm 9,5$ Jahre, $\mathrm{FEV}_{1} 1,23 \pm 0,69 \mathrm{l}$, entsprechend $41,59 \pm 19,51 \%$ Soll). Eingeschlossen wurden Patienten mit einer klinisch manifesten Hypoxämie mit einem arteriellen Sauerstoffpartialdruck $\leq 55 \mathrm{~mm} \mathrm{Hg}$ unter Raumluft in Ruhe $\left(\mathrm{PaO}_{2} 48,8 \pm 6,7 \mathrm{~mm} \mathrm{Hg}\right)$. Ausgeschlossen wurden Patienten mit akuter Exazerbation der chronisch obstruktiven Atemwegserkrankung. Alle Patienten befanden sich in einem stabilen Zustand ihrer Erkrankung. Sie atmeten während des Tests Sauerstoff über eine Sauerstoffbrille (Fa. Kendall bei D und $\mathrm{P}$, Fa. Weinmann bei $\mathrm{O}$ entsprechend Empfehlungen des Herstellers).

Bei den verwendeten Sauerstoff-Demandsystemen handelte es sich um DOC-2000 (TransTracheal Systems, Inc.) an einer Flüssigsauerstoffquelle, Oxytron II (Weinmann) an einer Sauerstoffdruckflasche und Pulsair (DeVilbiss, Sunrise Medical) an einer Flüssigsauerstoffquelle.

Die drei verwendeten Demandsysteme arbeiten zwar alle nach einem ähnlichen Prinzip, jedoch unterscheiden sie sich in der Art der Sauerstoff-Freisetzung. Gemeinsam ist den Systemen, dass ein elektronischer Drucksensor den subatmosphärischen Druck zu Beginn der Inspiration registriert, worauf sich ein Ventil öffnet und ein Sauerstoffbolus abgegeben wird.

Bei Pulsair ist das Volumen des frühinspiratorischen Sauerstoffbolus abhängig von der Öffnungsdauer des Sparventils. Das Ventil öffnet sich mit variabler Dauer, so öffnet es sich z.B. bei einer Einstellung äquivalent zu $21 / \mathrm{min}$ (nach Herstellerangaben) für eine Zeitdauer von $200 \mathrm{~ms}$ und es werden $33,0 \mathrm{ml}$ Sauerstoff freigesetzt.

Anders als Pulsair kann Oxytron nur einen Bolus mit einem festen Volumen von $35 \mathrm{ml}$ über einen Zeitraum von $200 \mathrm{~ms}$ freisetzen. Es kann eingestellt werden, ob ein solcher Sauerstoffbolus von $35 \mathrm{ml}$ nur bei einer von vier Inspirationen, zwei von vier, drei von vier oder bei jeder Inspiration abgegeben werden soll. Dies entspricht laut Herstellerangaben den Einstellungen äquivalent zu 1, 2, 3 oder 4 l/min unter kontinuierlichem Fluss. Eine Einstellung, bei der sich das Ventil bei jeder zweiten Inspiration öffnen würde, entspräche also einem kontinuierlichen Fluss von 2 1/min.

DOC-2000 setzt bei jeder Inspiration einen Sauerstoffbolus frei. Im Unterschied zu Pulsair wird jedoch bei einer festen Öffnungsdauer von $900 \mathrm{~ms}$ die abgegebene Sauerstoffmenge über ein variierendes Flussprofil verändert. Die Menge des freigesetzten Sauerstoffs ist äquivalent zu dem SauerstoffFluss, der an dem Regler der vorgeschalteten Sauerstoffquelle eingestellt ist. Bei einer Einstellung der Fluss-Reglers der Sauerstoffquelle auf $2 \mathrm{l} / \mathrm{min}$ wird also bei jeder Inspiration das Ventil für 0,9 Sekunden geöffnet und Sauerstoff strömt in dieser Zeit mit einer tatsächlichen Flussgeschwindigkeit von 2 l/min (oder anders ausgedrückt 33,33 ml/s) aus, was einem
Bolus von $33,33 \mathrm{ml} / \mathrm{s} \times 0,9 \mathrm{~s}=30 \mathrm{ml}$ entspricht. Ein solcher Bolus bei jeder Inspiration wäre folglich nach Herstellerangaben äquivalent zu 2 l/min mit kontinuierlichem SauerstoffFluss.

Aufgrund der unterschiedlichen Funktionsweise der Demandsysteme wird also je nach verwendetem Gerät ein unterschiedlich großer Sauerstoffbolus pro Inspiration freigesetzt. Bei einer Einstellung äquivalent zu 21/min kontinuierlicher Sauerstoffgabe (nach den jeweiligen Herstellerangaben) werden bei Pulsair pro Inspiration 33,0 ml Sauerstoff abgegeben, bei Oxytron sind es durchschnittlich $17,5 \mathrm{ml}$ (35,0 $\mathrm{ml}$ bei jeder zweiten Inspiration), bei DOC-2000 lässt sich ein BolusVolumen von $30,0 \mathrm{ml}$ pro Inspiration berechnen.

Die Applikation des Sauerstoffs erfolgte in randomisierter Reihenfolge mit jedem System für jeweils 30 Minuten. Die Messungen für die verschiedenen Systeme wurden dabei jeweils bei einem Patienten am gleichen Tag direkt aufeinander folgend ausgeführt und fanden unter Ruhebedingungen statt. Der Fluss-Regler war dabei auf $21 / \mathrm{min}$ eingestellt, beziehungsweise auf ein Äquivalent zu 21/min an dem jeweiligen Sparsystem entsprechend der Herstellerangaben.

Nach jeweils 15 und 30 Minuten wurden bei jedem System Blutproben aus dem hyperämisierten Ohrläppchen entnommen und die Blutgase bestimmt (Analysegerät AVL Omni, AVL Systems). Außerdem wurde die Sauerstoffsättigung kontinuierlich mit Hilfe eines Pulsoxymeters (Invivo 4500, Invivo Inc.) gemessen.

Des Weiteren galt es, den Spareffekt der einzelnen Demandsysteme zu quantifizieren, und damit den Reichweitengewinn eines gegebenen Sauerstoffvorrates zu ermitteln. Sämtliche Messungen zur Reichweitenbestimmung fanden ebenfalls unter Ruhebedingungen statt. Hierzu wurde zunächst eine Titration des $\mathrm{PaO}_{2}$-Wertes durchgeführt: Nach einer initialen Gabe von kontinuierlichem Sauerstoff mit einem Fluss von 21/min über einen Zeitraum von 30 Minuten wurde eine Blutprobe aus dem hyperämisierten Ohrläppchen entnommen und eine Blutgasanalyse durchgeführt. Danach benutzten die Patienten ein Demandsystem. Nach 30 Minuten wurden wieder die Blutgase bestimmt. Lag der $\mathrm{PaO}_{2}$-Wert unter dem Wert, der bei der Verwendung von kontinuierlichem Sauerstoff gemessen wurde, so wurde der Fluss-Regler des Demandsystems um eine Stufe höher gestellt und der Test wurde wiederholt. Dieser Schritt wurde so oft wiederholt, bis eine äquivalente Einstellung zum kontinuierlichen Fluss von $2 \mathrm{l} / \mathrm{min}$ bestimmt wurde, die die beste Annäherung des gemessenen $\mathrm{PaO}_{2}$-Wertes an den von kontinuierlichem Sauerstoff ergab.

Danach wurden die Patienten mit einem tragbaren Flüssigsauerstoffbehälter mit 0,5 I Inhalt (entsprechend 4001 Sauerstoff unter Atmosphärendruck) ausgestattet. Zunächst wurde der Sauerstoff kontinuierlich appliziert mit einem Fluss von $2 \mathrm{l} / \mathrm{min}$. Es wurde die Zeit gemessen, bis der Sauerstoff im Behälter vollständig aufgebraucht war. Danach wurde die gleiche Menge Flüssigsauerstoff in Verbindung mit DOC-2000 und der per Titration ermittelten Fluss-Einstellung gegeben. Erneut wurde die Zeit gemessen, bis der Sauerstoff vollständig aufgebraucht war. Die Messung mit Pulsair wurde analog durchgeführt. 
Für Oxytron erfolgte die Sauerstoffgabe aus einer Druckflasche mit 21 komprimiertem Sauerstoff (ebenfalls entsprechend 4001 Sauerstoff unter Atmosphärendruck). Auch hier wurde die Zeit gemessen, bis der Sauerstoff aus der Druckflasche unter kontinuierlicher Gabe mit $21 / \mathrm{min}$ und unter Verwendung von Oxytron mit der per Titration ermittelten Einstellung vollständig aufgebraucht war.

Zur statistischen Analyse wurde die Varianzanalyse mit nichtverbundenen Stichproben als lineares Modell verwandt.

\section{Ergebnisse}

Unter Raumluft hatten die Patienten einen arteriellen Sauerstoffpartialdruck von $48,8 \pm 6,7 \mathrm{~mm} \mathrm{Hg}$.

Nach 15 Minuten unter kontinuierlicher Sauerstoffgabe stieg der arterielle Sauerstoffpartialdruck auf $63,2 \pm 12,1 \mathrm{~mm} \mathrm{Hg}$ (Abb.1). Der $\mathrm{PaO}_{2}$-Wert für das Pulsair-System lag nach 15 Minuten bei $63,2 \pm 13,7 \mathrm{~mm} \mathrm{Hg}$, für DOC-2000 bei $59,1 \pm 14,4 \mathrm{~mm} \mathrm{Hg}$ und für Oxytron bei $58,7 \pm 9,0 \mathrm{~mm} \mathrm{Hg}$. Die Unterschiede erreichten jedoch keine Signifikanz.

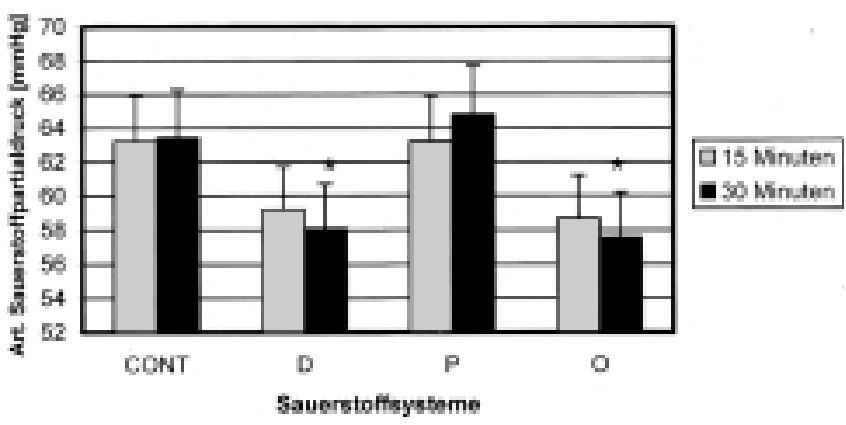

Abb. 1 Arterieller Sauerstoffpartialdruck nach 15 und 30 Minuten Anwendung der verschiedenen Sauerstoff-Demandsysteme $\left({ }^{*} \mathrm{p}<0,05\right.$ im Vergleich zu CONT). $\mathrm{D}=\mathrm{DOC}-2000, \mathrm{P}=$ Pulsair, $\mathrm{O}=\mathrm{Oxy}-$ tron, CONT = kontinuierliche Sauerstoffgabe.

Die $\mathrm{PaO}_{2}$-Werte erhöhten sich in der Phase mit kontinuierlicher Sauerstoffgabe nach 30 Minuten auf 63,5 $\pm 10,9 \mathrm{~mm} \mathrm{Hg}$. Es fand sich kein signifikanter Unterschied bezüglich des arteriellen Sauerstoffpartialdrucks zwischen der kontinuierlichen Sauerstoffgabe und dem Pulsair-System $\left(\mathrm{PaO}_{2}\right.$ $64,8 \pm 16,6 \mathrm{~mm} \mathrm{Hg}$ ). Der Sauerstoffpartialdruck war unter Verwendung des Pulsair mit dem inspirationsgetriggerten Demand-Flow nach 30 Minuten Anwendungszeit sogar geringfügig höher als unter kontinuierlicher Sauerstoffgabe. Signifikant erniedrigt hingegen waren die 30-Minuten-Werte des arteriellen Sauerstoffpartialdrucks für DOC-2000 $\left(\mathrm{PaO}_{2}\right.$ $58,1 \pm 11,7 \mathrm{~mm} \mathrm{Hg} ; \quad \mathrm{p}<0,01)$ und für Oxytron $\left(\mathrm{PaO}_{2}\right.$ $57,6 \pm 9,9 \mathrm{~mm} \mathrm{Hg} ; \mathrm{p}<0,01)$. Diese Signifikanzen ergaben sich auch beim Vergleich dieser Systeme mit dem Pulsair-System.

Darüber hinaus konnte im Gegensatz zu kontinuierlicher Sauerstoffgabe und Pulsair unter Verwendung von DOC-2000 bzw. Oxytron bei einer Enstellung äquivalent zu 2 1/min nach Herstellerangaben der arterielle Sauerstoffpartialdruck nicht in den therapeutisch erwünschten Bereich > $60 \mathrm{~mm} \mathrm{Hg}$ angehoben werden.
Die Sauerstoffsättigungswerte (Abb. 2) waren analog zu den Ergebnissen des Sauerstoffpartialdrucks, zusätzlich zeigten sich die signifikanten Unterschiede bei DOC-2000 und Oxytron im Vergleich zur kontinuierlichen Sauerstoffgabe bereits nach 15 Minuten.

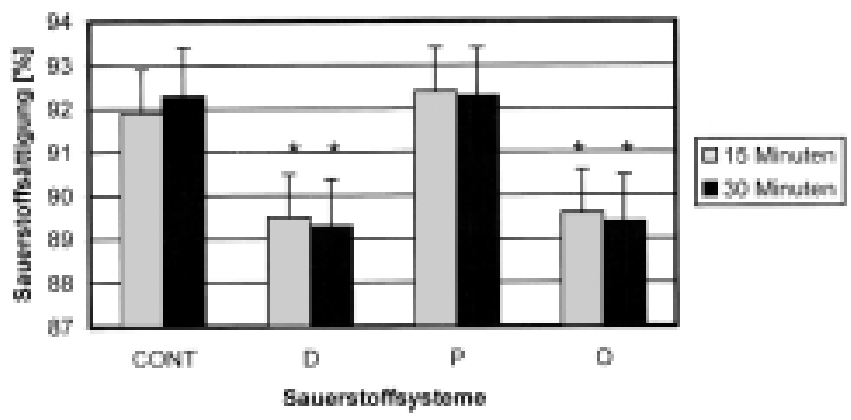

Abb. 2 Pulsoxymetrisch gemessene Sauerstoffsättigung nach 15 und 30 Minuten Anwendung der verschiedenen Sauerstoff-Demandsysteme ( ${ }^{*} \mathrm{p}<0,05$ im Vergleich zu CONT). $\mathrm{D}=\mathrm{DOC}-2000, \mathrm{P}=$ Pulsair, $\mathrm{O}=$ Oxytron, CONT = kontinuierliche Sauerstoffgabe.

Es zeigte sich, dass bei Verwendung von DOC-2000 ein höherer Sauerstoff-Fluss verwandt werden musste als bei kontinuierlicher Sauerstoffgabe, um den bei kontinuierlichem Fluss erreichten arteriellen Sauerstoffpartialdruck zu halten. Die Titration ergab, dass der Demand-Flow mit DOC-2000 äquivalent $\mathrm{zu} 3 \mathrm{l} / \mathrm{min}$ nach Herstellerangaben eingestellt werden musste $\left(\mathrm{PaO}_{2} 64,6 \pm 11,4 \mathrm{~mm} \mathrm{Hg}\right)$, um eine gute Annäherung an den unter $21 / \mathrm{min}$ kontinuierlicher Sauerstoffgabe gemessenen $\mathrm{PaO}_{2}$-Wert $(67,0 \pm 8,9 \mathrm{~mm} \mathrm{Hg})$ zu erreichen.

Bei Pulsair hingegen musste die Demand-Flowrate nicht angepasst werden. Die vom Hersteller gemachten Äquivalenzangaben stimmten mit den Ergebnissen der Titration überein. Mit einem Demand-Flow äquivalent zu 2 l/min nach Herstellerangaben hatten die Patienten einen $\mathrm{PaO}_{2}$ von $69,2 \pm 8,0 \mathrm{~mm} \mathrm{Hg}$ im Vergleich zu $67,0 \pm 8,9 \mathrm{~mm} \mathrm{Hg}$ bei kontinuierlicher Gabe von 2 l/min.

Bei der Titration mit Oxytron war der Ausgangswert der arterielle Sauerstoffpartialdruck unter kontinuierlicher Sauerstoffgabe von $21 / \mathrm{min}$, diesmal aus einer Sauerstoff-Druckflasche $(67,8 \mathrm{~mm} \mathrm{Hg})$. Auch hier musste der Demand-Flow äquivalent $\mathrm{zu} 3 \mathrm{l} / \mathrm{min}$ nach Herstellerangaben eingestellt werden. Damit betrug der arterielle Sauerstoffpartialdruck $66,7 \mathrm{~mm} \mathrm{Hg}$.

Die Reichweitenmessungen (Abb.3) wurden mit den zuvor per Titration ermittelten Äquivalenz-Flussraten durchgeführt. Zunächst wurden die Reichweiten bei kontinuierlicher Sauerstoffgabe und mit DOC-2000 verglichen. Der Flüssigsauerstoff-Vorrat von 0,51 war unter kontinuierlicher Sauerstoffgabe von 2 l/min im Durchschnitt nach 200,5 $\pm 3,5$ Minuten aufgebraucht. Unter Verwendung von DOC-2000 ließ sich die Reichweite auf 235,0 \pm 5,0 Minuten verlängern, was einem Reichweitenzuwachs von 17,2 Prozent entspricht.

Der Spareffekt beim Pulsair-System fiel wesentlich höher aus. Bei kontinuierlicher Sauerstoffgabe von 21/min war der Sauerstoffvorrat nach $195 \pm 3,6$ Minuten verbraucht. Unter 


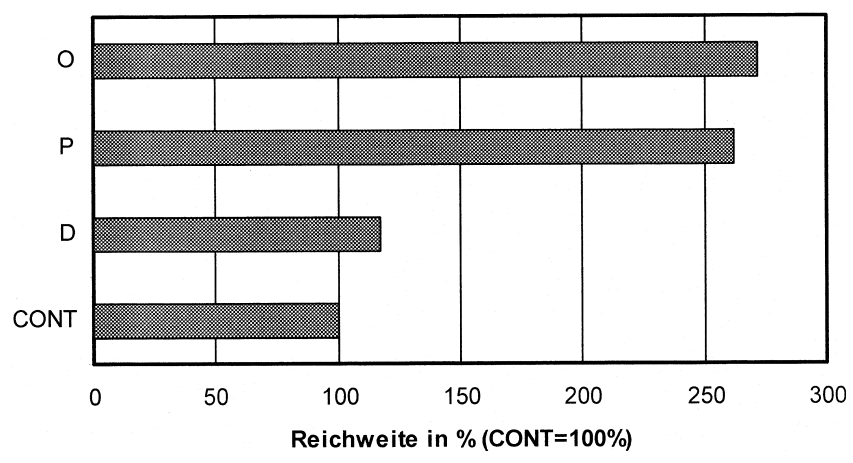

Abb. 3 Vergleich der Reichweite in \% (kontinuierliche Sauerstoffgabe $=100 \%$ ) bei kontinuierlicher Gabe von $2 \mathrm{l} / \mathrm{min}$ und bei Verwendung der Sauerstoff-Demandsysteme. $D=D O C-2000, P=$ Pulsair, $\mathrm{O}=$ Oxytron, CONT = kontinuierliche Sauerstoffgabe.

Verwendung von Pulsair waren es 510,0 \pm 13,5 Minuten. Dies entspricht 261,5 Prozent im Vergleich zu kontinuierlicher Sauerstoffgabe mit einem Fluss von 2 1/min.

Der größte Reichweitenzuwachs ließ sich mit Oxytron erzielen. Bei kontinuierlicher Gabe von 2 1/min reichte die Sauerstoff-Druckflasche mit einem Volumen von 21 für 200 Minuten. Mit Oxytron waren es 544 Minuten, und dies, obwohl der Demand-Flow erhöht werden musste (äquivalent zu $3 \mathrm{l} / \mathrm{min}$ nach Herstellerangaben). Die Reichweite vergrößerte sich auf 272 Prozent im Vergleich zu kontinuierlicher Sauerstoffgabe.

\section{Diskussion}

Aufgrund der unterschiedlichen Funktionsweise der Demandsysteme wird je nach verwendetem Gerät ein unterschiedlich großer Sauerstoffbolus pro Inspiration freigesetzt (bei einer Einstellung äquivalent $\mathrm{zu} 2 \mathrm{l} / \mathrm{min}$ laut Herstellerangaben bei Pulsair $33 \mathrm{ml}$, bei DOC-2000 $30 \mathrm{ml}$ und bei Oxytron durchschnittlich $17,5 \mathrm{ml}$ Sauerstoff pro Inspiration).

Dieser Sachverhalt erklärt die Tatsache, dass das PulsairSystem bei einem Fluss äquivalent zu 21/min wesentlich effizienter arbeitet als Oxytron bei einem Fluss äquivalent zu 2 1/min, denn pro Inspiration gibt Pulsair fast doppelt so viel Sauerstoff ab wie Oxytron. Selbst bei einer Erhöhung des Demand-Flow auf ein Äquivalent zu 3 l/min nach Herstellerangaben gibt Oxytron nur durchschnittlich 26,25 ml Sauerstoff pro Inspiration ab (je $35,0 \mathrm{ml}$ bei drei von vier Inspirationen), dies reicht aber aus, um einen Effekt zu erzielen, der mit kontinuierlichen Sauerstoffgabe von 2 1/min vergleichbar ist.

Anders verhält es sich bei DOC-2000. Hier werden bei einer Einstellung äquivalent $\mathrm{zu} 2 \mathrm{l} / \mathrm{min}$ nach Herstellerangaben $30,0 \mathrm{ml}$ Sauerstoff pro Inspiration abgegeben. Damit sollten vergleichbare Ergebnisse erzielt werden wie Pulsair bei einer Einstellung äquivalent $\mathrm{zu} 2 \mathrm{l} / \mathrm{min}(33,0 \mathrm{ml}$ pro Inspiration) oder mit Oxytron bei einer Einstellung äquivalent zu 3 l/min (26,25 $\mathrm{ml}$ pro Inspiration). In der Titration zeigte sich aber, dass dies nicht der Fall ist. Auch am DOC-2000-System musste der Fluss erhöht werden (äquivalent zu 3 1/min nach Herstellerangaben), um ein Ergebnis vergleichbar mit kontinuierlicher Sauerstoffgabe zu erreichen. Das pro Inspiration freigesetzte Bolusvolumen beträgt bei dieser Einstellung
45,0 ml. Wie der Vergleich der Reichweiten zeigt, geht dieses große Bolusvolumen deutlich zulasten der Reichweite.

Die schlechten Ergebnisse mit DOC-2000 beruhen wahrscheinlich auch auf einer $\mathrm{zu}$ hohen Triggerschwelle des Gerätes. Daraufhin deutet die Tatsache, dass der Apnoe-Alarm des DOC-2000 (Apnoe länger als 20 Sekunden) trotz normaler nasaler Atmung bei 15 von 17 Patienten mindestens einmal ausgelöst wurde. Außerdem fiel bei allen Patienten auf, dass das Gerät bei einigen Inspirationsvorgängen nicht triggerte und somit auch kein Sauerstoff abgegeben wurde. Eine höhere Triggerschwelle kann bedeuten, dass die Latenzzeit zwischen Inspirationsbeginn und Öffnen des Sparventils größer wird, so dass der Sauerstoffbolus verspätet abgegeben wird und somit frühinspiratorisch nur Raumluft geatmet wird. Das hieße wiederum, dass der Anteil des Sauerstoffbolus, der tatsächlich den Alveolarraum erreicht, vermindert ist, zumal bei DOC2000 der Bolus über einen verhältnismäßig langen Zeitraum von $900 \mathrm{~ms}$ abgegeben wird (im Vergleich zu $200 \mathrm{~ms}$ bei Pulsair).

In den Studien [2-4], welche die Effektivität verschiedener Sauerstoffsysteme untersuchen, wurde lediglich die pulsoxymetrisch gemessene Sauerstoffsättigung untersucht. In dieser Arbeit wurden zusätzlich Blutgasanalysen durchgeführt. Hierbei wurde deutlich, dass sich mit beiden Methoden die beschriebenen Unterschiede zwischen den Sauerstoff-Demandsystemen nachweisen lassen. Klinisch ist jedoch die Sauerstoffsättigung, die den Sauerstoffgehalt des Blutes besser repräsentiert, der relevantere Parameter.

Die Ergebnisse bezüglich des Spareffektes mit Pulsair und Oxytron stehen im Einklang mit den Ergebnissen, die in der Literatur zu finden sind. Für Oxytron wird sogar eine Vergrößerung der Reichweite auf das Siebenfache der Reichweite unter kontinuierlicher Sauerstoffgabe beschrieben [5,6]. Dabei wurde allerdings keine Titration durchgeführt. Wie in der vorliegenden Arbeit gezeigt wurde, ist eine Titration jedoch erforderlich. Daraus resultiert, dass der Sauerstoff-Fluss angepasst werden muss und dass der Spareffekt weniger deutlich ausgeprägt ist. Mit Oxytron kann dadurch lediglich noch ein Reichweitenzuwachs auf das 2,7fache erzielt werden. Mit DOC-2000 war nur ein Reichweitenzuwachs auf das 1,17fache möglich.

\section{Schlussfolgerung}

Inzwischen gibt es eine Reihe unterschiedlicher SauerstoffDemandsysteme. In dieser Studie sollte exemplarisch anhand von drei Systemen gezeigt werden, dass große Unterschiede hinsichtlich der Effektivität möglich sind, die durch Unterschiede in technischen Details bedingt sind.

Es wurde auch deutlich, dass man unabhängig von den Herstellerangaben bezüglich der äquivalenten Flusseinstellungen im individuellen Fall eine Titration durchführen sollte. So kann eine Einstellung gefunden werden, die tatsächlich äquivalent $\mathrm{zu}$ dem benötigten kontinuierlichen SauerstoffFluss ist. Diese kann erheblich von den Herstellerangaben abweichen. Eine solche Titration sollte mindestens über eine halbe Stunde durchgeführt werden, da die Einstellung eines Gleichgewichts des arteriellen Sauerstoffpartialdrucks bei Patienten mit COPD langsam erfolgt $[7,8]$. 
Aus methodischen Gründen erfolgten die Untersuchungen unter Ruhebedingungen. Unter Belastung ist eine Anpassung der Flussrate erforderlich und es sind noch größere Unterschiede zwischen den Systemen zu erwarten.

Der Einsatz von Sauerstoff-Demandsystemen ist unter Auswahl des richtigen Systems und korrekter Anwendung nicht nur geeignet, den Patienten eine größtmögliche Mobilität zu bieten, sondern es ist auch eine adäquate Methode zur Sauerstoffeinsparung.

\section{Literatur}

${ }^{1}$ Tiep BL, Carter R, Nicotra B, Berry J, Phillips RE, Otsap B. Demand oxygen delivery during exercise. Chest 1987; 91: 15-20

${ }^{2}$ Rinow ME, Saltzman AR. Effectiveness of a new oxygen demand valve in chronic hypoxemia. Chest 1986; 90: 204-207

${ }^{3}$ Haber H, Raber W, Kapfhammer G, Vetter N. Vergleich des Sauerstoff-Sparmoduls „Oxytron“ und der Reservoirbrille „Oxymizer Pendant“ mit kontinuierlicher Sauerstoffzufuhr über Nasenbrille bei hypoxämischen Patienten. Wien Klin Wochenschr 1990; 102: $325-329$

${ }^{4}$ Braun SR, Spratt G, Scott GC, Ellersieck M. Comparison of six oxygen delivery systems for COPD patients at rest and during exercise. Chest 1992; 102: 694-698

${ }^{5}$ Tiep BL, Nicotra MB, Carter R, Phillips R, Otsap B. Low-concentration oxygen therapy via a demand oxygen delivery system. Chest 1985; 87: 636-638

${ }^{6}$ Carter R, Tashkin D, Djahed B, Hathaway E, Nicotra MB, Tiep BL. Demand oxygen delivery for Patients with restrictive lung disease. Chest 1989; 96: 1307-1311

${ }^{7}$ Codes JE. Lung function assessment and application in medicine. Oxford: Blackwell Scientific Publications, 1997: 134-165

${ }^{8}$ Rogers RM, King TKC, Briscoe WA. Respiratory intensive care. Springfield: Charles C Thomas Publisher, 1977: 130-142
Dr. med. U. Johann

Universitätskliniken des Saarlandes Innere Medizin V

66421 Homburg / Saar 